NEUROSCIENCE FOR NEUROLOGISTS

Cerebral perfusion and stroke

H S Markus

J Neurol Neurosurg Psychiatry 2004;75:353-361. doi: 10.1136/jnnp.2003.025825

Stroke is a heterogeneous syndrome caused by multiple disease mechanisms, but all result in a disruption of cerebral blood flow with subsequent tissue damage. This review covers the mechanisms responsible for regulation of the normal cerebral circulation, and how they are disrupted in disease states. A central concept in treating patients with acute ischaemic stroke is the existence of an ischaemic penumbra of potentially salvageable tissue, and the evidence for its existence in humans is reviewed.

T he cerebral circulation has developed several specialised features to cope with the high metabolic demands of the brain and the devastating consequences of cerebral ischaemia. Unlike other organs, the brain uses glucose almost exclusively as its sole substrate for energy metabolism. As it is unable to store energy it requires a constant supply of oxygenated blood containing an adequate glucose concentration to maintain its function and structural integrity. The brain oxygen requirement in the adult human accounts for about $20 \%$ of that of the whole body, for a relative size of only $2 \%$. Brain oxygen uptake is even higher in children in the first decade of life, where it may be up to $50 \%$ of the total body oxygen supply. ${ }^{1}$ This high metabolic demand is matched by a disproportionately large amount of the total cardiac output passing to the brain. Total cerebral blood flow (CBF) at rest is about $800 \mathrm{ml} / \mathrm{min}$, which is $15-20 \%$ of total cardiac output. ${ }^{2}$ Cerebral perfusion is a high flow, low pressure system with relatively preserved diastolic flow. This can be appreciated by comparing Doppler waveforms from cerebral vessels with systemic vessels; the ratio of diastolic to systolic flow is much higher for the cerebral circulation. Anatomical features and functional responses, including cerebral autoregulation, allow this high flow and provide protection against ischaemia.

Correspondence to: Professor Hugh Markus, Clinical Neuroscience, St George's Hospital Medical School, Cranmer Terrace, London SW17 ORE, UK; h.markus@sghms.ac.uk

Received: 13 August 2003 Revised:

24 November 2003

Accepted:

28 November 2003

\section{CEREBROVASCULAR ANATOMY}

The cerebral circulation has a well developed collateral circulation which plays an important protective role. Of crucial importance are communications between the basal cerebral arteries at the circle of Willis. ${ }^{3}$ Natural variation in its integrity will determine whether carotid occlusion results in massive hemispheric stroke or is asymptomatic. Collateral supply may also occur through anastomoses between the external carotid artery branches and the intracerebral circulation, and there is a well developed plexus of anastomoses between cerebral vessels on the surface of the brain. ${ }^{4}$ Despite this collateral supply, there are regions of the brain that are particularly vulnerable to a fall in perfusion pressure. Such border zone areas include those at the boundaries between the anterior and middle and the middle and posterior cerebral arteries, and the "internal" border zone areas in the corona radiata and centrum semiovale. Territories supplied by the penetrating or perforating arteries that supply the white matter and subcortical grey matter nuclei are particularly vulnerable to ischaemia. These arteries are end arteries and therefore ischaemia in their territory results in more rapid cell death.

\section{METHODS FOR MEASUREMENT OF CEREBRAL BLOOD FLOW}

Various different techniques (table 1) allow measurement of regional cerebral perfusion, although many are only semiquantitative. Such techniques require a tracer or contrast agent and an imaging technique allowing its concentration to be measured. Commonly used techniques include positron emission tomography (PET), single photon emission computed tomography (SPECT), xenon computed tomography (CT) and contrast CT perfusion, and magnetic resonance imaging (MRI) perfusion studies.

\section{Nuclear medicine methods}

Nuclear medicine methods use radionucleides as tracers and the tomographic approach in image reconstruction. These can be distinguished on the basis of the physical characteristics of the radionucleides. SPECT uses low energy, photon emitting radionucleides. PET uses coincidence radiation employing positron emitting radionucleides which, after annihilation, produce two $511 \mathrm{keV} \tau$ rays. PET allows quantification, while with SPECT only semiquantification is possible. Both involve exposure to significant radiation doses. The tracer most commonly used for PET CBF measurements is ${ }^{15} \mathrm{O}$ labelled water. Only PET allows measurement of oxygen utilisation and therefore the oxygen extraction fraction

Abbreviations: $A D C$, apparent diffusion coefficient; AMPA, $\alpha$-amino-3-hydroxy-5-methyl-4-isoxazole; CBF, cerebral blood flow; CBV, cerebral blood volume; $\mathrm{CMRO}_{2}$, cerebral metabolic rate of oxygen; CPP, cerebral perfusion pressure; CVR, cerebrovascular resistance; DWI, diffusion weighted imaging; eNOS, endothelial nitric oxide synthase; IMP, inosine 5'-monophosphate; iNOS, inducible nitric oxide synthase; $M T T$, mean transit time; NMDA, N-methyl-D-aspartate; $\mathrm{nNOS}$, neuronal nitric oxide synthase; NOS, nitric oxide synthase; OEF, oxygen extraction fraction; PET, positron emission tomography; SPECT, single photon emission computed tomography; ${ }^{99 m} \mathrm{TcHMPAO}$, technetium-99m

hexamethylpropylene amine oxime 
Table 1 Methods of measurement of cerebral blood flow

\begin{tabular}{|c|c|c|}
\hline Technology & Specific methods & Important points \\
\hline \multicolumn{3}{|l|}{ Perfusion (regional CBF) } \\
\hline Positron emission tomography & ${ }^{15} \mathrm{O}$ labelled water & $\begin{array}{l}\text { Allows oxygen consumption } \\
\text { measurement } \\
\text { Quantitative }\end{array}$ \\
\hline Xenon based techniques & $\begin{array}{l}\text { Stable xenon methods } \\
\text { Intravenous xenon methods } \\
\text { Xenon SPECT } \\
\text { Xenon CT }\end{array}$ & Potentially quantitative \\
\hline MR based techniques & $\begin{array}{l}\text { Exogenous perfusion } \\
\text { Endogenous perfusion }\end{array}$ & $\begin{array}{l}\text { Using gadolinium bolus } \\
\text { Non-invasive }\end{array}$ \\
\hline CT based techniques & $\begin{array}{l}\text { Contrast perfusion imaging } \\
\text { Xenon CT }\end{array}$ & \\
\hline SPECT & $\begin{array}{l}99 \mathrm{~m} \text { TcHMPAO } \\
123 \text { l labelled IMP }\end{array}$ & Semiquantitative \\
\hline Transcranial Duplex ultrasound & Ultrasound perfusion & Quantification not yet possible \\
\hline $\begin{array}{l}\text { Volume flow (in major arteries) } \\
\text { Carotid and vertebral ultrasound } \\
\text { Transcranial Doppler ultrasound }\end{array}$ & $\begin{array}{l}\text { Doppler based techniques } \\
\text { Non-Doppler based techniques }\end{array}$ & $\begin{array}{l}\text { Flow in major arteries and not tissue } \\
\text { perfusion } \\
\text { Velocity rather than flow measurement }\end{array}$ \\
\hline \multicolumn{3}{|c|}{ 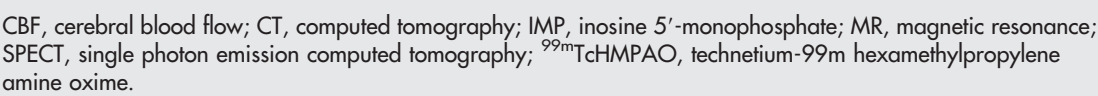 } \\
\hline
\end{tabular}

(OEF). SPECT is more widely available and uses lipophilic tracers that easily cross the blood-brain barrier and ideally are fully extracted during the first pass through the cerebral circulation. ${ }^{99 \mathrm{~m}}$ TcHMPAO is most widely used. It is stable for about six hours after being reconstituted, making it convenient for clinical use. A good correlation with CBF values has been obtained. ${ }^{5}$ Xenon is commonly used in cerebral perfusion measurement both with CT and SPECT. Xenon gas can be inhaled in significant quantities with little risk, although at high concentrations it is an anaesthetic. It dissolves into blood and readily diffuses through the bloodbrain barrier. Xenon has a high atomic number relative to air and soft tissues and as a result its presence can be measured by CT. A washout method can be used; when an initial equilibrium of substance has been reached in the tissue and the supply is acutely discontinued, the substance concentration decays in an exponential fashion and the decay of the exponential contains information on perfusion. ${ }^{6}$

\section{Computed tomography}

Perfusion CT involves sequential acquisition of stationary cerebral CT sections during intravenous administration of a bolus of iodinated contrast material. ${ }^{7}$ Analysis of the resultant contrast enhancement curves according to the central volume principle allows calculation of cerebral blood volume (CBV), mean transit time (MTT), and CBF.

\section{Magnetic resonance imaging}

Increasingly, MRI methods are used to measure perfusion. These fall into two categories.

Exogenous contrast methods are most widely used and rely on an intravenous bolus injection of a paramagnetic tracer and acquisition of rapid images, using echo-planar imaging, as the tracer passes through the cerebral vasculature. ${ }^{8}$ The effect of the tracer is to reduce signal from tissue in its immediate vicinity owing to dephasing effects caused by the magnetic field gradients induced. This signal loss is closely related to the flow of the tracer through the brain and thus to cerebral perfusion. This method also offers the ability to measure CBV and MTT. Its advantage is that paramagnetic substances have a large effect on signal intensity, and therefore the signal to noise ratio is high. Recent studies have suggested that a degree of quantification is possible if an arterial input function is obtained by imaging through a supplying vessel, ${ }^{8}$ although whether absolute quantification is reliable is debated.

Endogenous methods such as arterial spin labelling use blood water as an endogenous contrast agent for the measurement of perfusion. This is completely non-invasive and can be repeated as often as desired, but the signal to noise ratio is low.

\section{Ultrasound}

Ultrasound methods are non-invasive. Carotid and vertebral neck ultrasound uses either Doppler based ${ }^{9}$ or non-Doppler based methods ${ }^{10}$ to quantify volume flow in the major supplying arteries, rather than regional perfusion. In contrast, transcranial Doppler, most commonly of the middle cerebral artery, measures velocity and not flow and therefore only gives an accurate estimate of changes in flow if the diameter of the vessel being insonated does not change. Tissue perfusion can now be estimated using transcranial imaging ultrasound, but quantification using this technique is still being developed. ${ }^{11}$

\section{REGULATION OF THE NORMAL CEREBRAL CIRCULATION}

\section{Basal cerebral blood flow regulation}

Under normal conditions CBF is determined by both cerebral perfusion pressure (CPP) and cerebrovascular resistance (CVR). CVR is determined by the diameter of the intracranial arteries and also blood viscosity. CPP can be calculated from the difference between systemic arterial pressure and venous back pressure. In conditions were CPP remains constant any change in CBF must result from a change in CVR, usually as a result of alteration in the diameter of the small intracranial arteries, which are the predominant resistance vessels. Thus under normal circumstances there is a direct correlation between CBF and cerebral blood volume, which will both increase as vessels dilate and decrease as vessels constrict. This relation is disturbed in pathological states such as cerebral ischaemia.

Various physiological variables influence $\mathrm{CBF}$, including arterial blood gases, cerebral autoregulation, and metabolic rate through vasoneuronal coupling. One important mediator controlling basal CBF is nitric oxide. In $1980^{12}$ it was shown that an intact endothelial cell layer is required if isolated arterial segments were to relax in response to perfusion with 
acetylcholine. It was subsequently found that this "endothelium derived relaxing factor" is nitric oxide (NO), synthesised by endothelial cells from the amino acid L-arginine by the enzyme NO synthase (NOS). ${ }^{13}$ There are three isoforms of this enzyme: endothelial NOS (eNOS), neuronal NOS (nNOS), and an inducible NOS (iNOS). Endothelium derived NO plays a crucial role in maintenance of blood vessel calibre and therefore blood flow throughout the vasculature. It is also important in preventing thrombosis through inhibition of platelet adhesion, activation, and aggregation, and in preventing atherosclerosis by inhibition of vascular smooth muscle cell proliferation. Under normal conditions there appears to be a functional balance between the endothelium dependent vasodilator effects of $\mathrm{NO}$ and the endothelium derived constrictor substances such as endothelin, thromboxane, and angiotensin II. Extensive animal data in a variety of species have shown that tonic release of NO plays an important role in maintaining resting CBF. ${ }^{14}$ More recently the role of NO in maintaining basal CBF has been shown in the human: inhibition of NO by the NO synthase inhibitor Nmonomethyl-L-arginine (L-NMMA) resulted in a 30\% fall in CBF. ${ }^{15}{ }^{16}$

\section{Cerebral autoregulation}

CBF remains relatively constant despite moderate variations in perfusion pressure. This phenomenon, described as "cerebral autoregulation," plays an important protective role against the danger of hypoxia at low perfusion pressure, and the risk of brain oedema at higher arterial pressure. ${ }^{17}$ As a rough approximation the lower and upper limits of autoregulation occur at mean arterial pressures of 60 and 150 $\mathrm{mm} \mathrm{Hg}$ in the normotensive human. Between these limits $\mathrm{CBF}$ is relatively but not absolutely constant. Once the limits of autoregulation are reached, CBF increases or decreases passively with increases or reductions in perfusion pressure.

Reductions in CBF below the limit of autoregulation result in brain hypoperfusion. In an attempt to compensate for this, the extraction coefficient of oxygen from the blood increases. Clinical symptoms of functional disruption are not observed until the reduction in CPP exceeds the ability of the increase in oxygen extraction to satisfy the metabolic demands of cerebral tissues. If mean arterial pressure increases above the upper limit of autoregulation, resistance arteries in the brain cannot sustain vasoconstriction. An early sign is the appearance of "sausage stringing," characterised by an alternating pattern of dilated arterial segments with focal regions of constriction. The dilated segments represent regions of passive dilatation, and the constricted segments regions of sustained autoregulation. Further increases in CPP result in dilatation along the entire length of the arterioles, and CBF increases passively. This is accompanied by damage to the cerebrovascular endothelium and disruption of the blood-brain barrier. The latter results in extravasation of plasma proteins through the vessel wall and subsequent oedema. These events are important in acute hypertensive encephalopathy.

The upper and lower limits of autoregulation are not fixed but vary under both physiological stimuli and disease states. Activation of the sympathetic nerves results in upward shift of both the lower and upper limits-a potentially protective response because acute elevations in arterial pressure are usually accompanied by sympathetic activation. The autoregulatory plateau is shifted to higher values in patients with chronic hypertension. ${ }^{18}$ This protective response can have deleterious effects if blood pressure is excessively reduced, when symptoms of ischaemia may occur at a relatively higher blood pressure.

Cerebral autoregulation is impaired in various disease states including head injury, ${ }^{19}$ ischaemic stroke, $^{20}$ and subarachnoid haemorrhage. ${ }^{21}$ This may result in an already damaged brain being excessively sensitive to fluctuations in perfusion pressure.

Assessment of cerebral autoregulation in the human Traditionally, studies in the human have estimated "static" autoregulation. Steady state values of CBF are determined across a range of blood pressures. This is achieved by the use of drugs or shifts in blood volume-for example, using tilt tables or negative pressure. ${ }^{22}$ Determination of the full range of autoregulation requires blood pressure to be manipulated across a wide range, and the long time interval between measurements made at different pressures can make interpretation of studies difficult. More recently methods for estimating "dynamic" autoregulation in humans have been developed. These determine the rate of response of CBF or flow velocity after a sudden change in arterial blood pressure. A technique allowing measurement of CBF with very high temporal resolution is required. The only technique that can provide this is transcranial Doppler ultrasound. In the most widely used technique a sudden fall in blood pressure is induced by inflating leg cuffs above venous pressure and then rapidly deflating them. ${ }^{23}$ The rate of rise of CBF velocity in the middle cerebral artery is then compared with the rate of rise of blood pressure continuously monitored using a noninvasive device such as a Finapres. Using this technique, autoregulation has been shown to be impaired distal to carotid artery stenosis, ${ }^{24}$ following head injury, ${ }^{25}$ and in ischaemic stroke. ${ }^{26}$ Although the measurement of dynamic autoregulation gives a useful estimate of how the cerebral circulation can compensate for fluctuations in perfusion pressure, it may not necessarily be measuring the same mechanisms that determine static autoregulation.

\section{Physiological mechanisms of autoregulation}

The mechanisms responsible for CBF autoregulation in the human are not fully understood. Traditional hypotheses are that neurogenic, myogenic, and metabolic factors play a role. More recently NO has been implicated. Autoregulation is preserved in animals that have undergone sympathetic and parasympathetic denervation, suggesting that neurogenic factors are not of primary importance. ${ }^{27}$

The myogenic hypothesis states that smooth muscle in the resistance arteries responds directly to alterations in perfusion pressure by contracting during increases in pressure, and relaxing during reductions in pressure. This would provide a mechanism whereby responses in cerebral resistance arteries could occur within seconds of an autoregulatory stimulus. In vitro animal experiments support this hypothesis, with constriction of isolated arteries occurring following a rapid increase in intravascular pressure. In vivo experiments have not, in general, provided such strong support. ${ }^{27}$ The metabolic hypothesis states that reductions in CBF stimulate the release of vasoactive substances from the brain, which in turn stimulates the dilatation of cerebral resistance arteries. Several candidates for this role have been proposedincluding carbon dioxide, hydrogen ions, oxygen, adenosine, potassium, and calcium-but no definite role has been demonstrated for any of these. ${ }^{28}$

Endothelial factors, particularly NO, have been suggested as mediators of cerebral autoregulation. In some animal studies, impaired autoregulation has been reported following inhibition of NO, but others have found no effect. ${ }^{29}$ In eNOS knockout mice there was a greater fall in CBF during haemorrhagic hypotension than in wild type mice, consistent with an alteration in the lower limit of autoregulation. ${ }^{30}$ In humans the NOS inhibitor L-NMMA resulted in a significant impairment of dynamic autoregulation. ${ }^{29}$ 
Arterial blood gases, the hypercapnic response, and CBF

$\mathrm{CBF}$ is very sensitive to changes in blood $\mathrm{CO}_{2}$ concentrations, largely mediated by concomitant changes in the $\mathrm{pH}$ of brain tissue. ${ }^{31}$ Apart from this direct effect of $\mathrm{pH}$ on cerebral vessels, secondary mechanisms may mediate vasoactive effects through a pH dependent alteration in the release of other vasoactive factors. Prostaglandins have been suggested as mediators of $\mathrm{CO}_{2}$ dependent changes in $\mathrm{CBF}$, based on the effects of the inhibitor of cyclo-oxygenase, indomethacin. However, further studies in animals ${ }^{31}$ and humans ${ }^{32}$ cast doubt on this mechanism in adults, although it does appear to be important in newborn animals. ${ }^{31}$

Studies in rats suggested NO dependence of the hypercapnic response, although this NO production is primarily through nNOS rather than eNOS. However, results in higher species have been less consistent, and studies in primates have shown conflicting results. ${ }^{33} 34$ A complicating factor in animal studies is that anaesthesia may alter cerebrovascular responses. A study in humans showed that inhibition of eNOS by intravenous $\mathrm{N}^{\mathrm{G}}$-monomethyl-L-arginine (L-NNMA) did not alter the hypercapnic response. ${ }^{15}$

Reducing arterial $\mathrm{PO}_{2}$ results in an increase in $\mathrm{CBF}$, whereas an increase in arterial $\mathrm{PO}_{2}$ above normal values has a much lesser effect. Hypoxia appears to act both through a direct effect on cerebral resistance vessels, and through indirect effects including the release of vasoactive factors such as hydrogen ions, adenosine, and potassium ions. ${ }^{31}$ It has also been suggested that $\mathrm{pH}$ dependent mechanismsmainly the production of lactate-participate in hypoxic vasodilatation. ${ }^{35}$

\section{Clinical relevance of the hypercapnic response}

The response of CBF to hypercapnia is widely used in humans to estimate cerebral perfusion reserve, most often in patients with carotid stenosis or occlusion. The haemodynamic consequences of a carotid stenosis-for example, during a fall in blood pressure-will depend on distal collateral supply, particularly through the circle of Willis. An estimate of this cerebral perfusion reserve can be obtained by measuring CBF during both normocapnia and hypercapnia (with concentrations of $5-8 \% \mathrm{CO}_{2}$ in air). In the presence of impaired reserve, resistance vessels are already vasodilated, and the capacity to increase CBF further is reduced (fig 1). A wide range of imaging methods has been used to measure the hypercapnic response, including PET, SPECT, MRI, and xenon based techniques. For techniques that do not allow absolute quantification (such as SPECT), side to side differences are calculated; this makes interpretation difficult in bilateral

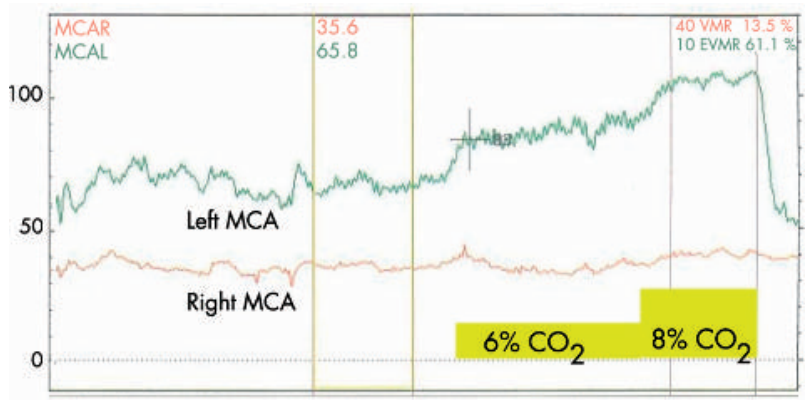

Figure 1 Measurement of cerebral perfusion reserve or cerebral reactivity in a patient with unilateral right internal carotid artery occlusion. Blood flow velocity is recorded continuously from both middle cerebral arteries. As the patient breaths a $6 \%$ and then an $8 \%$ mixture of carbon dioxide in air, flow velocity increases on the left but not on the right. This indicates severely impaired perfusion reserve ipsilateral to the occlusion. MCA, middle cerebral artery. carotid stenosis. Transcranial Doppler monitoring of middle cerebral artery flow velocity has become widely adopted in this context. ${ }^{36}$ Its validity is suggested by angiographic studies showing that the diameter of the middle cerebral artery does not change during hypercapnia. ${ }^{37}$ Acetazolamide, a carbonic anhydrase inhibitor, can be used as the vasodilator stimulus instead of $\mathrm{CO}_{2}$.

Prospective studies have shown that a reduced hypercapnic response ipsilateral to a carotid occlusion is associated with an increased risk of stroke or transient ischaemic attacks (TIA) ${ }^{38-40}$ It has been suggested this test may identify a subgroup of patients with carotid occlusion who benefit from extracranial-intracranial bypass, although this hypothesis has yet to be tested. There is some evidence from prospective studies that patients with tight carotid stenosis, as opposed to occlusion, and who have impaired perfusion reserve are at increased risk of stroke, but the association appears less strong than for carotid occlusion. ${ }^{39}$

\section{Local coupling of cerebral blood flow and metabolism} Local CBF is tightly coupled to changes in neuronal metabolism, and this forms the basis of functional brain imaging using BOLD MRI. Over a century ago Roy and Sherrington suggested that "...(the brain) is well fitted to provide for a local variation of the blood supply in accordance with local variations of the functional activity" ${ }^{41}$ Numerous studies have shown that local increases in neuronal activity result in a local increase in glucose utilisation, accompanied by local increases in CBF. The precise mechanisms linking neuronal activation, metabolism, and flow are not fully understood. Various factors are likely to contribute to this coupling process, including potassium release with neuronal depolarisation, and $\mathrm{H}^{+}$and adenosine release when there is a mismatch between oxygen delivery and utilisation. NO may play a key mediator role, probably through production via nNOS rather than eNOS. In animal models, cerebral vasodilatation associated with simple somatosensory stimulation appears to be mediated by nNOS derived $\mathrm{NO}^{42}$ In the human, L-NMMA given intravenously did not alter the local CBF response to learned sequential movements, but, because of its poor blood-brain barrier penetration, it probably only inhibits eNOS. ${ }^{16}$

\section{CEREBRAL CIRCULATION RESPONSES TO FOCAL ISCHAEMIA}

Protective responses to a progressive fall in cerebral perfusion pressure

CPP can fall because of systemic arterial hypotension, or severe stenosis in an extracranial or intracranial supplying artery, or a combination of the two. As CPP falls, intracranial resistance vessels dilate to maintain $\mathrm{CBF}$; this results in an increase in CBV. When vasodilatation is maximal, further falls in CPP result in a fall in CBF. Because oxygen delivery to the brain normally greatly exceeds demand, metabolic activity is maintained initially by increasing the OEF from blood. When oxygen extraction becomes maximal, flow is inadequate to meet metabolic demands, cellular metabolism is impaired, and the cerebral metabolic rate of oxygen $\left(\mathrm{CMRO}_{2}\right)$ begins to fall. This sequence of events is illustrated in fig 2 .

\section{Critical flow thresholds}

As cerebral autoregulation is impaired or lost in moderate to severe ischaemia, CBF varies passively with CPP. This relation has allowed investigators to gradually reduce CBF and assess critical flow thresholds at which certain functions are lost. ${ }^{43}$ Experimental studies in primates ${ }^{44}$ and cats, ${ }^{45}$ and clinical studies in humans during carotid endarterectomy, ${ }^{46}$ have shown that spontaneous and evoked electrical activity ceases 


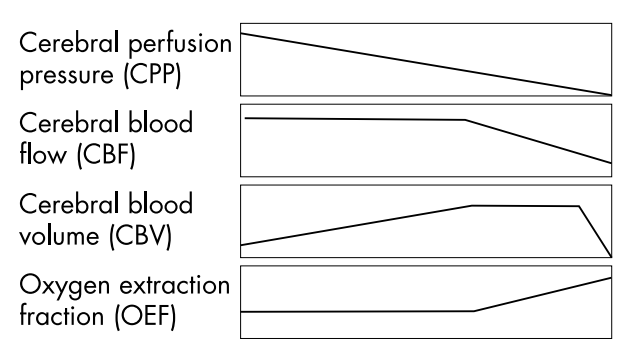

Figure 2 A schematic illustration of the consequences of a progressive fall in cerebral perfusion pressure. This can occur because of a progressive fall in arterial blood pressure, or because of a stenosis or occlusion of a major supplying artery to the brain. As CPP falls, CBF is maintained by vasodilatation. This results in a progressive rise in CBV. When vasodilatation is maximal, cerebral autoregulation is exhausted, and a further reduction in CPP results in a decrease in CBF. As CBF falls, oxygen extraction is increased and functional consequences of reduced $\mathrm{CBF}$ only occur when OEF is maximal.

when CBF falls below $16-18 \mathrm{ml} / 100 \mathrm{~g} / \mathrm{min}$. This level of ischaemia therefore represents a threshold for loss of neuronal electrical function (that is, electrical failure). It was subsequently shown that there is a lower threshold (10$12 \mathrm{ml} / 100 \mathrm{~g} / \mathrm{min}$ ) for loss of cellular ion haemostasis (that is, membrane failure). At this lower threshold, $\mathrm{K}^{+}$is released from and $\mathrm{Ca}^{2+}$ taken up by the cells. ${ }^{47}$ Rapid efflux of $\mathrm{K}^{+}$and uptake of $\mathrm{Ca}^{2+}$ represents a generalised collapse of membrane function and at this point cells also take up $\mathrm{Na}^{+}$and $\mathrm{Cl}^{-}$with osmotically obligated water. ${ }^{43}$ The threshold for infarction appears similar to that for energy failure/loss of membrane haemostasis, but it varies with the duration of the insult.

More recent studies suggest that the pattern of thresholds may be more complex, although the general principle of two major critical flow thresholds (loss of electrical function and then loss of cellular ion haemostasis) still applies. ${ }^{48}$ Protein synthesis is inhibited first (at a CBF of about $50 \mathrm{ml} / \mathrm{l} 00 \mathrm{~g}$ / $\mathrm{min}$ ), and is completely suppressed below $35 \mathrm{ml} / 100 \mathrm{~g} / \mathrm{min}$. This is above the level at which glucose utilisation and energy metabolism are disrupted. Glucose utilisation transiently increases at flow rates below $35 \mathrm{ml} / 100 \mathrm{~g} / \mathrm{min}$, before it sharply declines below $25 \mathrm{ml} / 100 \mathrm{~g} / \mathrm{min}$. This corresponds to anaerobic glycolysis with the beginning of acidosis and the accumulation of lactate. At flow rates below $26 \mathrm{ml} / 100 \mathrm{~g} / \mathrm{min}$, tissue acidosis becomes pronounced and both phosphocreatine and ATP begin to decline. ${ }^{48}$ Anoxic depolarisation, as assessed by recording extracellular potassium and calcium activities, occurs at even lower values $(<15 \mathrm{ml} / 100 \mathrm{~g} / \mathrm{min})$ (fig 3).

\section{THE ISCHAEMIC PENUMBRA}

The concept of an ischaemic penumbra is crucial to the treatment of acute stroke. It follows on from the finding of separate thresholds for electrical failure and loss of ion haemostasis. The concept is that following a focal ischaemic insult a penumbral region exists around a core of densely ischaemic and irreversibly damaged tissue. This penumbral region contains electrically inexcitable but viable cells. It follows that if perfusion is restored to this region, tissue salvage may be possible. The concept originated in the mid 1970s from experiments done on baboons. ${ }^{49}$ After middle cerebral artery occlusion, brain tissue could be identified which had lost its electrical function but did not show the release of potassium that characterises cell death. This functionally silent tissue was nevertheless viable because it regained its function and did not undergo necrosis if flow was restored early enough. This tissue was named the ischaemic penumbra. Topographically the penumbra was shown to be mainly cortical surrounding the ischaemic core,

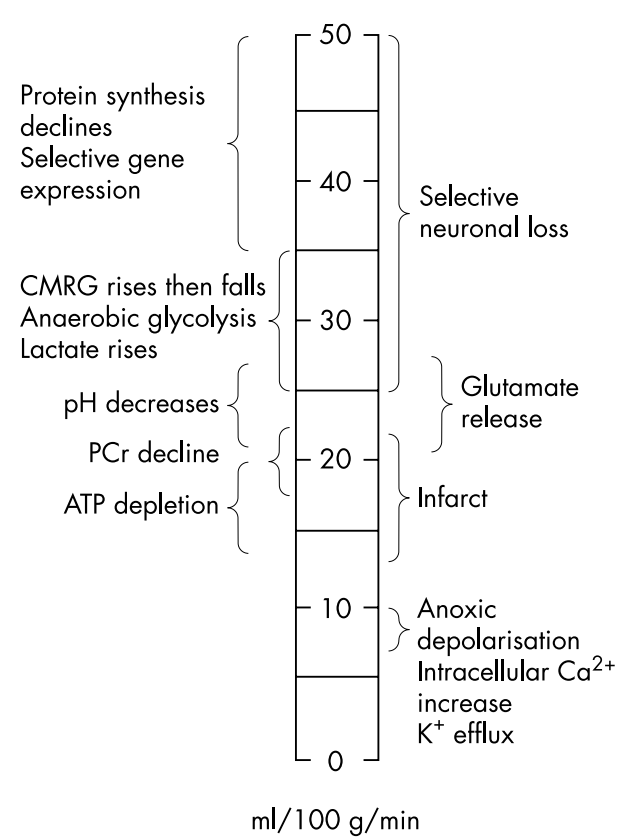

Figure 3 Thresholds of ischaemia for the induction of functional, metabolic, and histological lesions. Exact levels vary slightly in different animal models and also with the duration of ischaemia for certain variables. CMRG, cerebral metabolic rate of glucose; $\mathrm{PCr}$, phosphocreatine. Modified from Hossman, ${ }^{48}$ fig 1.

which in the baboon, as in the human, predominantly involves the subcortical structures supplied by the perforating arteries. In later studies it was found that the duration of ischaemia, as well as the absolute flow, plays a crucial role in determining the fate of ischaemic tissue. For example, in the macaque monkey, tissue with CBF of around $15 \mathrm{ml} / 100 \mathrm{~g} /$ min could withstand about three hours of occlusion, while tissue with a perfusion of around $5 \mathrm{ml} / 100 \mathrm{~g} / \mathrm{min}$ would stand only two hours. ${ }^{50}$ This time dependence has crucial significance when considering acute treatment in humans, and implies that treatment will be most successful when it is given as early as possible. A crucial question when assessing the potential for acute stroke therapy is how extensive the ischaemic penumbra is in the human.

\section{Is there an ischaemic penumbra in the human?}

PET studies have suggested that there is a significant ischaemic penumbra in humans, and that reversibly ischaemic tissue may persist for much longer than initial experiments in smaller animals suggested. ${ }^{51}$ These studies used oxygen-PET and quantitatively measured the main physiological variables involved in tissue ischaemia-namely $\mathrm{CBF}$, $\mathrm{CMRO}_{2}, \mathrm{OEF}$, and $\mathrm{CBV}$. Using this technique, a pattern of changes termed "misery perfusion" can be identified. This is characterised by reduced CBF with relatively preserved or even normal $\mathrm{CMRO}_{2}$, thus fitting the concept of penumbra with tissue of reduced perfusion but relatively maintained neuronal function. The hallmark of misery perfusion is an increased OEF, ranging from the normal value of about 30$40 \%$ up to a theoretical maximum of $100 \%$, where the tissue extracts all the oxygen supplied by the blood flow reaching it.

In the late 1980s and early 1990s several groups applied PET to investigate the pathophysiology of ischaemic stroke. In the acute phase in patients with anterior circulation stroke, misery perfusion was found to affect mainly the cortex, while the capsule-basal ganglia region often showed markedly reduced $\mathrm{CMRO}_{2}$, and only moderately increased OEF. ${ }^{52}{ }^{53}$ Deterioration of $\mathrm{CMRO}_{2}$ from the acute to subacute 
stage of stroke was reported, and this was interpreted as the transition from ischaemia to necrosis. However, no study directly documented the presence of tissue that was still metabolically active within a region that went on to infarction, as determined by follow up CT or MRI. ${ }^{51}$

Baron and colleagues studied 30 patients with first ever middle cerebral artery territory ischaemia within 18 hours of stroke onset and co-registered these early PET scans with late CT scans done to assess final infarct size. ${ }^{54}$ Those voxels or regions within the finally infarcted tissue with preserved $\mathrm{CMRO}_{2}$ on acute stage PET were identified. Of the 30 patients, there were eight who survived until the late CT, had follow up PET, had a middle cerebral artery territory infarct of $>16$ $\mathrm{ml}$ as measured on late CT scanning, and had technically adequate PET studies. In seven of these, voxels were found which progressed to infarction but initially had $\mathrm{CMRO}_{2}$ values above the threshold for irreversible damage. The tissue identified represented substantial volumes of the final infarct, being a mean of $35 \%$ and as high as $52 \%$ in one patient. Furthermore "penumbral" tissue was observed in two cases at 16 hours and in another case at 13 hours after stroke onset.

Proving that this is indeed penumbral tissue requires intervention studies in which the penumbra is salvaged by treatment that restores flow. However, the concept is supported by the results of clinical trials showing that thrombolysis improves outcome, ${ }^{55}$ and also by more recent MRI studies (see below). Further support is provided by PET studies in the baboon which showed that tissue with acute misery perfusion went on to infarction if the middle cerebral artery was permanently occluded, but escaped infarction if the artery was reopened at six hours. ${ }^{56}$ Interestingly, the time window for reperfusion in the baboon was much longer than in previous macaque experiments, ${ }^{48}$ and considerably longer than PET studies in the cat. ${ }^{57}$ These significant species differences emphasise the importance of undertaking studies in humans.

\section{Can MRI identify an ischaemic penumbra?}

Although PET studies give quantitative information on CBF and oxygen metabolism they are not widely available, difficult to do in large numbers of patients, and involve the administration of radioisotopes. The ability to identify potentially salvageable tissue using more widely available techniques has great attraction. There is considerable interest in the potential of MRI to identify an "ischaemic penumbra," based on a combination of perfusion and diffusion weighted imaging techniques. Most studies have used exogenous perfusion techniques to measure the perfusion deficit. The most promising technique for identifying ischaemic tissue is diffusion weighted imaging (DWI). Diffusion imaging is dependent on the apparent diffusion coefficient (ADC) of water in tissue. Following ischaemia, ADC values rapidly decline, corresponding to a region of high signal on DWI. This ADC decline is likely to reflect the accumulation of intracellular water, cytotoxic oedema, disruption of high energy metabolism, and loss of ion homeostasis. These ADC changes do not occur uniformly in the ischaemic region, and in experimental stroke models ischaemic tissue with the most severe perfusion deficit has the earliest and most severe fall in ADC. Serial DWI studies in animal models have demonstrated the evolution of ADC changes over time, and shown that mild declines in ADC early after stroke are potentially reversible.

The concept of diffusion-perfusion mismatch has been developed as a possible way of identifying potentially salvageable tissue in the human. The rationale is that tissue that is abnormal on DWI and has low perfusion is destined for infarction. In contrast, tissue that is normal on DWI but has perfusion reduced to penumbral levels may either recover, particularly if flow is restored, or progress to infarction. There is considerable support for this hypothesis. Within six hours of stroke onset approximately $70 \%$ of acute patients will have a perfusion volume greater than the diffusion volume, $20 \%$ will have equal volumes, and in $10 \%$ the perfusion volume will be smaller, representing spontaneous reperfusion. ${ }^{58}$ Baird et al studied 13 patients with ischaemic stroke in whom both DWI and perfusion imaging were measured at an initial time point (2 to 53 hours) and at follow up time points ( 7 to 725 days)..$^{59}$ Lesion volume increased by $230 \%$ when the perfusion volume exceeded the initial DWI volume, but decreased by $47 \%$ when perfusion volume was smaller than or equivalent to the DWI volume. Barber et al reported an increase of $62 \%$ in the DWI lesion volume between acute ( $<24$ hours) and subacute (3-5 days) time points, when the perfusion volume was greater than the DWI lesion volume (11 of 17 patients). ${ }^{60}$ The remaining six patients in whom the perfusion volume was less than the DWI volume had a stable DWI lesion volume.

Studies in patients receiving thrombolysis provide further evidence that this mismatch does represent reversibly ischaemic tissue. Patients undergoing intravenous thrombolysis, who underwent successful reperfusion, had reduced lesion volume as defined by DWI and later by T2 weighted MRI. ${ }^{61}$ Similar results have been found in patients receiving intra-arterial thrombolytic treatment. ${ }^{62}$ MRI studies also emphasised the heterogeneity of human stroke. In some patients mismatch is still present many hours after stroke, consistent with the PET studies, ${ }^{51}$ while in others there is no mismatch early after stroke. This may partly explain why acute stroke trials have been disappointing and it has led to the suggestion that treatment needs to be tailored to the patient more effectively. Current therapeutic trials are targeting patients with persisting mismatch for treatment with thrombolytic and other agents.

Recent data have suggested that the diffusion-perfusion concept is more complex. Initially it was thought that tissue appearing abnormal on DWI in humans was almost always destined to infarction. Case reports were published showing reversibility of DWI changes, and a more recent series has confirmed that DWI abnormalities may recover, ${ }^{63}$ although probably in a minority of patients. Furthermore on repeat scanning at seven days such recovered areas may remain normal, or DWI and T2 abnormalities may reappear. This is consistent with animal studies showing secondary delayed ADC declines after temporary focal occlusion. The clinical significance of these changes is not fully understood, but if they predict a worse clinical outcome they may represent a therapeutic target for post-reperfusion neuroprotective targets. Despite the MR diffusion-perfusion concept being more complicated than initially appreciated, there is considerable evidence that it does allow "penumbral" tissue to be identified. Its clinical use will depend upon current prospective randomised trials showing that it can identify a subgroup of patients who benefit particularly from therapeutic interventions such as thrombolysis.

MRI is much more widely available than PET, but nevertheless few units can offer it to all patients with acute stroke. This has led to attempts to use the more available technology of CT perfusion to identify the extent of reversibly ischaemic tissue. Compared with DWI, non-contrast CT imaging is poor at showing ischaemic tissue in the first few hours. However, with the use of a rapid intravenous injection of iodinated contrast in combination with newer multislice CT scanners, maps can be obtained of CBF, CBV, and MTT, in addition to conventional non-contrast CT images, with acquisition times of less than 10 minutes. $^{7}$ CT perfusion measurements have been found to correlate well with stable 


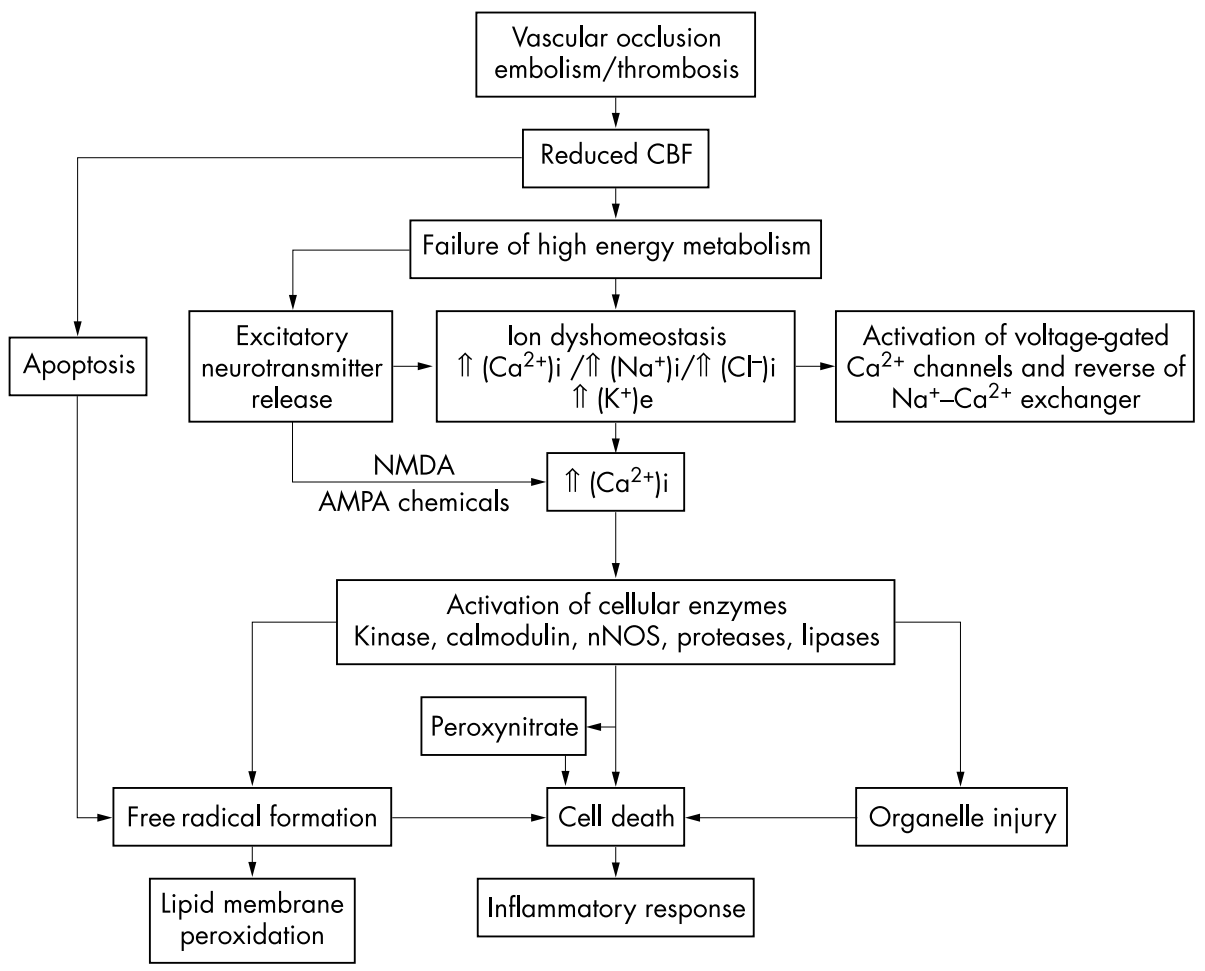

Figure 4 Mechanisms mediating tissue injury in focal cerebral ischaemia. Modified from fig 4.5 in Fisher, 2002. ${ }^{58}$ Multiple mechanisms which interact with each other are involved. This may partly explain why neuroprotective approaches inhibiting one pathway have failed in the treatment of human ischaemic stroke. AMPA, $\alpha$-amino-3-hydroxy-5-methyl-4-isoxazole; NMDA, N-methyl-D-aspartate; NNOS, neuronal nitric oxide synthase; (.....)i, intracellular; (.....)e, extracellular.

xenon-CT estimates. ${ }^{64}$ Using this method, an ischaemic cerebral area (penumbra plus irreversibly damaged tissue) has been defined as a $\mathrm{CBF}$ reduction of more than $34 \%$ compared with the contralateral hemisphere. ${ }^{65}$ Within this area, regions with a CBV below a predefined cut off $(2.5 \mathrm{ml} /$ $100 \mathrm{~g}$ ) were selected as irreversibly damaged tissue, with the remainder defined as penumbra. In a validation study against MRI in 13 subjects, a highly significant correlation was found between the CT defined irreversibly damaged tissue and the region of MR DWI abnormality. ${ }^{65}$ The ischaemic cerebral area correlated strongly with the MR defined MTT defect, but less well with the MRI perfusion defect obtained from CBF maps. The authors suggested that CT perfusion provides a similar degree of information to MRI on penumbral tissue. Other small studies have also shown correlations with MRI estimates, ${ }^{66}$ but much more data from larger series are required, ideally including comparisons with PET as well as with MRI. ${ }^{67}$ This should both confirm these findings, and show that the CT derived information allows prediction of outcome and selection of a group who may benefit from thrombolytic and other treatments. A further limitation of CT perfusion in comparison with MRI is that slice coverage is limited.

\section{MECHANISMS OF INFARCTION IN PENUMBRAL TISSUE}

A complex cascade of mechanisms is responsible for the progression of penumbral tissue to infarction. Understanding these processes is of crucial importance for developing potentially effective treatment strategies. Mechanisms determining both flow and the cellular and metabolic consequences of hypoperfusion are important.

Key factors determining outcome are the presence and extent of collateral flow, and the time at which recanalisation occurs. Cerebral autoregulation is disrupted within the ischaemic penumbra, which may make the tissue particularly vulnerable to alterations in blood pressure. Continued embolisation and thrombus propagation may also play a role $^{68}$ Experimental evidence suggests that progressive microvascular obstruction contributes to the progression of ischaemic damage following stroke. ${ }^{69}$ Various processes may contribute to the activation of cerebral microvessels following ischaemia, including alterations in integrin-matrix interactions, leucocyte endothelial cell adhesion, blood-brain barrier permeability changes, and microvascular occlusion owing to adhesion of leucocytes, activated platelets, and fibrin deposition. ${ }^{69}$

A complex cascade of cellular and metabolic consequences follows focal ischaemic injury. This is illustrated in fig 4 and has been reviewed in detail elsewhere. ${ }^{5870}$ Only some of the more important aspects are covered below.

Excitotoxicity appears to play a crucial role. ${ }^{71}$ Glutamate release activates postsynaptic $\mathrm{N}$-methyl-D-aspartate (NMDA) and $\alpha$-amino-3-hydroxy-5-methyl-4-isoxazole (AMPA) receptors. This results in calcium and sodium influx into cells, leading to cellular oedema and activation of the catabolic processes that destroy cellular integrity. These initial events are amplified by further increases in intracellular calcium through activation of voltage mediated calcium channels. ${ }^{72}$ The intracellular calcium increase induces protein kinases and calmodulin regulated enzymes. Glutamate release appears to be a primary process in a cascade of molecular reactions mediated by NO and free radicals. NO generated in neurones and microglia causes cell death by inhibiting mitochondrial functions, including apoptosis, and promoting the formation of free radicals such as the highly toxic peroxynitrite radicals. Knockout mice which lack nNOS develop infarcts of smaller size. ${ }^{73}$ Glutamate diffusion from areas with high concentration and more severe ischaemia to less severely ischaemic regions may also lead to progression 
of injury. Cerebrospinal fluid and plasma concentrations of glutamate are higher in patients with progressing ischaemic stroke than in those with non-progressing stroke. ${ }^{74}$

Peri-infarct depolarisations may play an important role in progression of damage. ${ }^{75}$ These depolarisations are similar to the phenomena of spreading depression observed after mechanical or chemical injury to normal cortical tissue in animal models. DWI studies of rat stroke models have shown that peri-infarct depolarisations lead to an increase in the size of the ischaemic lesion. ${ }^{76}$ The underlying mechanism may be an increase in energy demands upon already compromised tissue by this energy consuming process. ${ }^{58}$ In normal tissue, this increased energy demand is met by an increase in perfusion, but this is not possible when CBF is reduced. The relevance of peri-infarct depolarisations in human stroke has not been determined, although with DWI monitoring it may be possible to show an imaging correlate.

Inflammation is probably important in extending ischaemic injury. Most inflammatory reactions are mediated by cytokines. These have been implicated in several mechanisms that may potentiate ischaemic brain injury, ${ }^{77}$ including release of NO from inducible NOS by astrocytes, recruitment, activation, and adhesion to the endothelium of infiltrating leucocytes, the promotion of a local procoagulant state, and the regulation of apoptotic processes.

\section{SUMMARY}

Many anatomical and physiological responses ensure that the brain receives adequate blood supply, and protect it against the devastating consequences of cerebral ischaemia. The disruption of these by focal ischaemia can now be investigated by an ever increasing range of methods to estimate perfusion, many of which can be implemented on routine clinical neuroimaging equipment. PET studies in humans have demonstrated reversibly ischaemic penumbral tissue following focal ischaemia; its extent varies markedly between patients but in a subgroup it may persist for hours. It is hoped that imaging techniques will allow patients with salvageable tissue to be identified for specific therapeutic interventions.

Competing interests: none declared

\section{REFERENCES}

1 Nehlig A. Metabolism of the central nervous system. In: Mraovitch S, Sercombe R, eds. Neurophysiological basis of cerebral blood flow control: an introduction. London: John Libby, 1996:177-96.

2 Kety SS. Circulation and metabolism of the human brain in health and disease. Am J Med 1950;8:205-17.

3 Riggs HE, Rupp C. Variation in form of the circle of Willis. Arch Neurol 1963:8:8-14.

4 Vander Ecken HM, Adams RD. The anatomy and functional significance of the meningeal arterial anastamoses of the human brain. J Neuropathol Exp Neurol 1953; 12:132-57.

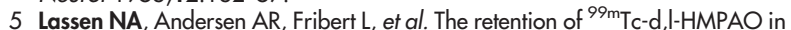
the human brain after intra-arterial bolus injection. A kinetic analysis. J Cereb Blood Flow Metab 1988;8:S13-22.

6 Yonas H, Pindzola RP, Johnson DW. Xenon/computed tomography cerebral blood flow and its use in clinical management. Neurosurg Clin North Am 1996:7:605-16.

7 Lev MH, Sagal AZ, Farkas J, et al. Utility of perfusion-weighted CT imaging in acute middle cerebral artery stroke treated with thrombolysis. Prediction of final infarct volume and clinical outcome. Stroke 2001;32:2021-8.

8 Ostergaard L, Johannsen P, Host-Poulsen P, et al. Cerebral blood flow measurements by magnetic resonance imaging bolus tracking: comparison with $[(15) \mathrm{O}] \mathrm{H}_{2} \mathrm{O}$ positron emission tomography in humans. J Cereb Blood Flow Metab 1998; 18:935-40.

9 Eicke BM, Tegeler $\mathrm{CH}$. Ultrasonic quantification of blood flow volume. In: Tegeler CH, Babikian VL, Gomez CR, eds. Neurosonology. St Louis: CV Mosby, 1995: 100-9.

10 Deane CR, Markus HS. Colour velocity flow measurement. In vitro validation and application to the human carotid arteries. Ultrasound Med Biol 1997:23:447-52.

11 Eyding J, Wilkening W, Postert T. Brain perfusion and ultrasonic imaging techniques. Eur J Ultrasound 2002;16:91-104

12 Furchcott RF, Zawadski JV. The obligatory role of endothelial cells in the relaxation of arterial smooth muscle by acetylcholine. Nature 1980;288:373-6.
13 Palmer RM, Ferrige AG, Moncada S. Nitric oxide release accounts for the biological activity of endothelium-derived relaxing factor. Nature 1987;327:524-6.

14 ladecola C, Pelligrino DA, Moskowitz MA, et al. Nitric oxide synthase inhibition and cerebrovascular regulation. J Cereb Blood flow Metab 1994; 14:175-92.

15 White RP, Deane C, Vallance P, et al. Nitric oxide synthase inhibition in humans reduces cerebral blood flow but not the hyperaemic response to hypercapnia. Stroke 1998;29:467-72.

16 White RP, Hindley C, Bloomfield PM, et al. The effect of the nitric oxide synthase inhibitor L-NMMA on basal CBF and vasoneuronal coupling in man: a PET study. J Cereb Blood Flow Metab 1999;19:673-8.

17 Lassen NA. Cerebral blood flow and oxygen consumption in man. Physiol Rev 1959;39:183-238.

18 Strandgaard S, Olsen J, Skinhoj E, et al. Autoregulation of brain circulation in severe arterial hypertension. BMJ 1973;1:507-10.

19 Enevoldsen EM, Jensen FT. Autoregulation and $\mathrm{CO}_{2}$ responses of cerebral blood flow in patients with acute severe head injury. I Neurosurg 1978;48:689-703

20 Symon L, Branston NM, Strong AJ. Autoregulation in acute focal ischaemia. An experimental study. Stroke 1976;7:547-54.

21 Voldby B. Pathophysiology of subarachnoid haemorrhage. Experimental and clinical data. Acta Neurochir Suppl (Wien) 1988;45:1-6.

22 Panerai RB. Assessment of cerebral pressure autoregulation in humans - a review of measurement methods. Physiol Meas 1998;19:305-38.

23 Tiecks FP, Lam AM, Aaslid R, et al. Comparison of static and dynamic cerebral autoregulation measurements. Stroke 1995;26:1014-19.

24 White RP, Markus HS. Non-invasive determination of impaired dynamic cerebral autoregulation in carotid artery stenosis. Stroke 1997;28:1340-4.

25 Hlatky R, Furuya Y, Valadka AB, et al. Dynamic autoregulatory response after severe head injury. J Neurosurg 2002;97:1054-61.

26 Dawson SL, Panerai RB, Potter JF. Serial changes in static and dynamic cerebral autoregulation after acute ischaemic stroke. Cerebrovasc Dis 2003:16:69-75

27 Busija DW, Heistad DD. Factors involved in the physiological regulation of the cerebral circulation. Rev Physiol Biochem Pharmacol 1984;101:161-211.

28 Chillon J-M, Baumbach GL. Autoregulation of cerebral blood flow. In: Welch KMA, Caplan LR, Reis DJ, et al, eds. Primer on cerebrovascular diseases. San Diego: Academic Press, 1997:51-4

29 White R, Vallance P, Markus HS. The effect of nitric oxide synthase inhibition on dynamic cerebral autoregulation in man. Clin Sci (Colch) 2000:99.555-60.

30 Huang Z, Huang PL, Ma J, et al. Enlarged infarcts in endothelial nitric synthase knockout mice are attenuated by nitro-l-arginine. J Cereb Blood Flow Metab 1996;16:981-7.

31 Kuschinsky W. Regulation of cerebral blood flow: an overview. In: Mraovitch S, Sercombe R, eds. Neurophysiological basis of cerebral blood flow control: an introduction. London: John Libby, 1996:245-62.

32 Markus HS, Vallance P, Brown MM. Differential effect of three cyclooxygenase inhibitors on human cerebral blood flow velocity and carbon dioxide reactivity. Stroke 1994;25:1760-4.

33 Thompson BG, Pluta RM, Girton ME, et al. Nitric oxide mediation of chemoregulation but not autoregulation of cerebral blood flow in primates. J Neurosurg 1996:84:71-8.

34 McPherson RW, Kirsch JR, Ghaly RF, et al. Effect of nitric oxide synthase inhibition on the cerebral vascular response to hypercapnia in primates. Stroke 1995;26:682-7

35 Shinozuka T, Nemoto EM, Winter PM. Mechanisms of cerebrovascular $\mathrm{O}_{2}$ sensitivity from hyperoxia in the rat. J Cereb Blood Flow Metab 1989:9: 187-95.

36 Ringlestein EB, Sievers $\mathrm{C}$, Ecker S, et al. Noninvasive assessment of $\mathrm{CO}_{2}$ induced cerebral vasomotor response in normal individuals and patients with internal carotid artery occlusions. Stroke 1988;19:963-9.

37 Huber $\mathbf{P}$, Handa J. Effect of contrast material, hypercapnia, hyperventilation, hypertonic glucose and papaverine on the diameter of cerebral arteries. Invest Radiol 1967;2:17-32.

38 Kleiser B, Widder B. Course of carotid artery occlusions with impaired cerebrovascular reactivity. Stroke 1992;23:171-4.

39 Markus $\mathrm{H}$, Cullinane M. Severely impaired cerebrovascular reactivity predicts stroke and TIA risk in patients with carotid artery stenosis and occlusion. Brain 2001; 124:457-67.

40 Gur AY, Bova I, Bornstein NM. Is impaired cerebral vasomotor reactivity a predictive factor of stroke in asymptomatic patients? Stroke 1996;27:2188-90

41 Roy CS, Sherrington CS. On the regulation of the blood supply of the brain. J Physiol (Lond) 1890;1 1:85-108.

42 Gjedde A. Brain energy metabolism and the haemodynamic response. In: Jezzard P, Matthews PM, Smith SM, eds. Functional MRI. Oxford: Oxford University Press, 2001:37-65.

43 Siesjo BK. Pathophysiology and treatment of focal cerebral ischaemia. Part 1. Pathophysiology. J Neurosurg 1992;77:169-84.

44 Branston NM, Symon L, Crockard HA, et al. Relationship between the cortical evoked potential and local cortical blood flow following acute middle cerebral artery occlusion in the baboon. Exp Neurol 1974:45:195-208.

45 Heiss WD, Hayakawa T, Waltz AG. Cortical neuronal function during ischaemia. Effects of occlusion of one middle artery on single unit activity in cats. Arch Neurol 1976;33:813-20.

46 Sharborough FW, Messick JM, Sundt TM. Correlation of continuous electroencephalograms with cerebral blood flow measurements during carotid endarterectomy. Stroke 1973;4:674-83. 
47 Harris RJ, Symon L, Branston NM, et al. Changes in extracellular calcium activity in cerebral ischaemia. J Cereb Blood Flow Metab 1981;1:203-9.

48 Hossmann KA. Viability thresholds and the penumbra of focal ischemia. Ann Neurol 1994:36:557-65.

49 Astrup J, Siesjo BK, Symon L. Thresholds in cerebral ischemia - the ischemic penumbra. Stroke 1981;12:723-5.

50 Jones TH, Moraweta RB, Crowell RM, et al. Thresholds of focal cerebral ischemia in awake monkeys. J Neurosurg 1981;54:773-82.

51 Baron JC. Mapping the ischaemic penumbra with PET: implications for acute stroke treatment. Cerebrovasc Dis 1999:9:193-201.

52 Baron JC, Bousser MG, Comar D, et al. Noninvasive tomographic study of cerebral blood flow and oxygen metabolism in vivo: Potentials, limitations and clinical applications to cerebral ischemic disorders. Eur Neurol 1981;20:273-84.

53 Wise RJS, Bernadi S, Frackowiak RSJ, et al. Serial observations on the pathophysiology of acute stroke: the transition from ischaemia to infarction as reflected in regional oxygen extraction. Brain 1983;106:197-222.

54 Marchal G, Beaudouin V, Rioux P, et al. Prolonged persistence of substantial volumes of potentially viable brain tissue after stroke: a correlative PET-CT study with voxel-based data analysis. Stroke 1996;27:599-606.

55 rt-PA Stroke Study Group. Tissue plasminogen activator for ischemic stroke. N Engl J Med 1995;333:1581-7.

56 Young AR, Touzani O, Derlon JM, et al. Early reperfusion in the anaesthetized baboon reduces brain damage following middle cerebral artery occlusion. Stroke 1997;28:632-8.

57 Heiss WD, Graf R, Wienhard K, et al. Dynamic penumbra demonstrated by sequential multitracer PET after middle cerebral artery occlusion in cats. J Cereb Blood Flow Metab 1994;14:892-902.

58 Fisher $M$. The ischemic penumbra and the therapeutic time window. In: Fisher M, Bogousslavsky J, eds. Current review of cerebrovascular disease, 4th ed. Philadelphia: Current Medicine, 2002:35-43.

59 Baird A, Benfield A, Schlaug G, et al. Enlargement of human cerebral ischemic lesion volumes measured by diffusion-weighted magnetic imaging. Ann Neurol 1997;41:581-9.

60 Barber P, Darby D, Desmond PM, et al. Prediction of stroke outcome with echoplanar perfusion- and diffusion-weighted magnetic resonance imaging Neurology, 1998;51;418-26.

61 Schellinger PD, Jansen O, Fiebach JB, et al. Monitoring intravenous recombinant tissue plasminogen activator thrombolysis for acute ischemic stroke with diffusion and perfusion MRI. Stroke 2000;31:1318-28.
62 Kidwell CS, Saver JL, Mattiello J, et al. Thrombolytic reversal of acute human cerebral ischemic injury shown by diffusion/perfusion magnetic resonance imaging. Ann Neurol 2000;47:462-9.

63 Kidwell CS, Saver JL, Starkman S, et al. Late secondary ischemic injury in patients receiving intra-arterial thrombolysis. Ann Neurol 2002;52:698-703.

64 Wintermark M, Maeder P, Thiran J-P, et al. Simultaneous measurements of regional cerebral blood flows by perfusion-CT and stable xenon-CT: a validation study. Am J Neuroradiol 2001;22:905-14.

65 Wintermark M, Reichhart M, Cuisenaire O, et al. Comparison of admission perfusion computed tomography and qualitative diffusion- and perfusionweighted magnetic resonance imaging in acute stroke patients. Stroke 2002;33:2025-31.

66 Na DG, Ryoo JW, Lee KH, et al. Multiphasic perfusion computed tomography in hyperacute ischemic stroke: comparison with diffusion and perfusion magnetic resonance imaging. J Comput Assist Tomogr 2003;27:194-206.

67 Warach S. Stroke neuroimaging. Stroke 2003;34:345-7.

68 Wong KS, Gao S, Chan YL, et al. Mechanisms of acute cerebral infarctions in patients with middle cerebral artery stenosis: a diffusion-weighted imaging and microemboli monitoring study. Ann Neurol 2002;52:74-81.

69 del Zoppo GJ, Hallenback JM. Advances in the vascular pathophysiology of ischemic stroke. Thromb Res 2000;98:V73-81.

70 Kogure T, Kogure K. Molecular and biochemical events within the brain subjected to cerebral ischemia. Clin Neurosci 1997;4:179-83.

71 Choi DW, Lobner D, Dugan LL. Glutumate receptor-mediated neuronal death in the ischemic brain. In: Hsu C, ed. Ischemic stroke: from basic mechanisms to new drug development. Basel: Karger, 1998:2-13.

72 Mody I, MacDonald JF. NMDA receptor-dependent excitotoxicity. The role of intercellular $\mathrm{Ca}^{++}$release. Trends Pharmacol Sci 1995;16:356-9.

73 Samdani AF, Dawson TM, Dawson VI. Nitric oxide synthase in models of focal ischaemia. Stroke 1997;28:1283-8.

74 Castillo J, Davalos A, Noya M. Progression of ischaemic stroke and excitotoxic aminoacids. Lancet 1997;349:79-83.

75 Hossman KA. Peri-infarct depolarizations. Cerebrovasc Brain Metab Rev 1996;8:195-208.

76 Takano K, Latour LL, Formato JE, et al. The role of spreading depression in focal ischemia evaluated by diffusion mapping. Ann Neurol 1996:39:308-18.

77 Vila N, Castillo, Davalos A, et al. Proinflammatory cytokines and early neurological worsening in ischemic stroke. Stroke 2000;31:2325-9. 